radiation-induced defects. This reduction in phonon scattering was a strong indication that the main phonon scattering was from mobile dislocations, rather than from their static strain fields. To further test these ideas, we $\gamma$ irradiated our dislocated sample with a Cs source and then compared the intensities of horizontal and vertical FTA ridges. With $0.6 \mathrm{Mrad}$ of $\gamma$ radiation the scattering strength $\beta$ was found to decrease by a factor of 7, shown as the dotted line in Fig. 3(c). With a 6-Mrad dose, the intensity of the vertical ridge was increased by an additional factor of 7 , as shown by the dashed line in Fig. 3(c). Thus we find that the $\gamma$ irradiation reduced the total scattering strength by nearly a factor of 50 . This large reduction in scattering of particular phonon polarizations with irradiation may be contrasted to the much smaller reduction in thermal conductivity, where many modes and polarizations are sampled.

Our experiments and analyses thus show that the method of ballistic phonon imaging yields new and interesting details about the scattering of high-frequency phonons from dislocations in LiF. The ability to quantitatively sample the phonon transmission as a continuous function of propagation angle and accurately identify the phonon polarization from the singularity pattern provides a powerful new approach to characterizing the interaction of phonons with defects in insulators.
We acknowledge the useful advice and suggestions of A. V. Granato. These experiments were supported in part by National Science Foundation Grant No. NSF-DMR-80-20250 and by U. S. Department of Energy Grant No. DE-AC02-76ER01198. The computer-controlled imaging system was developed under the National Science Foundation Grant No. DMR-80-24000.

${ }^{1}$ A. C. Anderson and M. E. Malinowski, Phys. Rev. B 5, $3199(1972)$; A. C. Anderson, in Dislocations in Solids, edited by F. R. N. Nabarro (North-Holland, New York, to be published).

${ }^{2}$ A. V. Granato, Phys. Rev. 111, 740 (1958); see also $T$. Ninomiya, in Treatises on Materials Science and Technology, edited by H. Herman (Academic, New York, 1975), Vol. 8; F. R. N. Nabarro, Theory of Crystal Dislocations (Oxford Univ. Press, Oxford, England, 1967), p. 505; A. V. Granato, in Internal Friction and Ultrasonic Attenuation in Crystalline Solids, edited by D. Lenz and K. Lücke (Springer-Verlag, New York, 1975), Vol. II, pp. 33-47.

${ }^{3} \mathrm{E}$. J. Cotts, D. M. Miliotis, and A. C. Anderson, Phys. Rev. B 24, 7336 (1981).

${ }^{4}$ G. A. Northrop and J. P. Wolfe, Phys. Rev. B 22 , 6196 (1980), and references therein.

${ }^{5}$ G. A. Kneezel and A. V. Granato, Phys. Rev. B 25 , 2851 (1982). We do not distinguish between single dislocation and dipoles here: $\Omega(\theta, \varphi)$ is the same for both.

\title{
Surface Studies by Scanning Tunneling Microscopy
}

\author{
G. Binning, H. Rohrer, Ch. Gerber, and E. Weibel \\ IBM Zurich Research Laboratory, 8803 Rüschlikon $-Z H$, Switzerland
}

(Received 30 April 1982)

\begin{abstract}
Surface microscopy using vacuum tunneling is demonstrated for the first time. Topographic pictures of surfaces on an atomic scale have been obtained. Examples of resolved monoatomic steps and surface reconstructions are shown for (110) surfaces of $\mathrm{CaIrSn}_{4}$ and $\mathrm{Au}$.

PACS numbers: $68.20 .+t, 73.40 . \mathrm{Gk}$
\end{abstract}

In two previous reports, ${ }^{1,2}$ we demonstrated the experimental feasibility of controlled vacuum tunneling. The tunnel current flowed from a W tip to a Pt surface at some $10 \AA$ distance from each other. The tunnel distance could be stabilized within $0.2 \AA$. These experiments were the first step towards the development of scanning tunneling microscopy. Previous developments were unsuccessful for various reasons. ${ }^{3}$
The present Letter contains the first experimental results on surface topography obtained with this novel technique. They demonstrate an unprecedented resolution of the scanning tunneling microscope (STM) and should give a taste of its fascinating possibilities for surface characterization.

The principle of the STM is straightforward. It consists essentially in scanning a metal tip 
over the surface at constant tunnel current as shown in Fig. 1. The displacements of the metal tip given by the voltages applied to the piezodrives then yield a topographic picture of the surface. The very high resolution of the STM rests on the strong dependence of the tunnel cur rent on the distance between the two tunnel electrodes, i.e., the metal tip and the scanned surface. The tunnel current through a planar tunnel barrier of average height $\psi$ and width $s$ is given by $^{4}$

$$
J_{T} \propto \exp \left(-A \psi^{1 / 2} s\right),
$$

where $A=(4 \pi / h) 2 m)^{1 / 2}=1.025 \AA^{-1} \mathrm{eV}^{-1 / 2}$, with $m$ the free-electron mass, appropriate for a vacuum tunnel barrier. With barrier heights (work functions) of a few electronvolts, a change of the tunnel barrier width by a single atomic step $(\sim 2-5 \AA)$ changes the tunnel current up to three orders of magnitude. Using only the distance dependence as given by Eq. (1), and a spherical tip of radius $R$, one estimates a lateral spread $\delta$ of a surface step as $\delta \approx 3 r_{0}=3\left(2 R / A \psi^{1 / 2}\right),{ }^{1 / 2}$ i.e., $\delta(\AA) \approx 3[R(\AA)]^{1 / 2}$. Thus, a lateral resolution considerably below $100 \AA$ requires tip radii of the order of $100 \AA$. Such tips are standard in field-

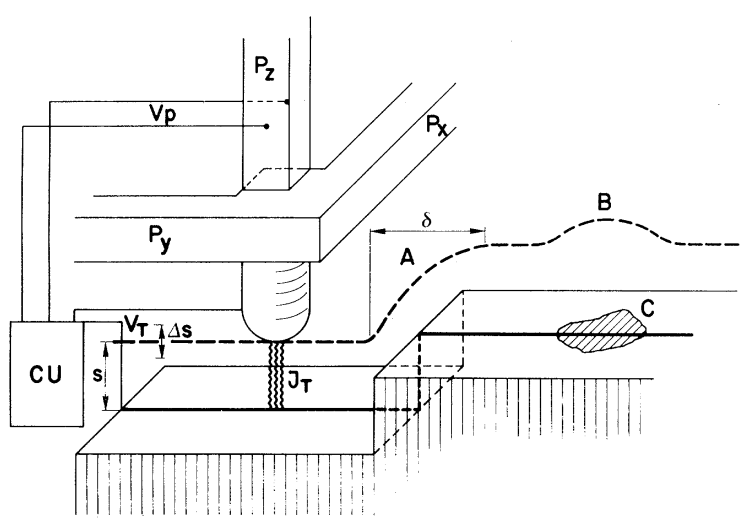

FIG. 1. Principle of operation of the scanning tunneling microscope. (Schematic: distances and sizes are not to scale.) The piezodrives $P_{x}$ and $P_{y}$ scan the metal tip $M$ over the surface. The control unit (CU) applies the appropriate voltage $V_{p}$ to the piezodrive $P_{z}$ for constant tunnel current $\boldsymbol{J}_{T}$ at constant tunnel voltage $V_{T}$. For constant work function, the voltages applied to the piezodrives $P_{x}, P_{y}$, and $P_{z}$ yield the topography of the surface directly, whereas modulation of the tunnel distance $s$ by $\Delta s$ gives a measure of the work function as explained in the text. The broken line indicates the $z$ displacement in a $y$ scan at $(A)$ a surface step and $(B)$ a contamination spot, $C$, with lower work function. emission microscopy. However, since suppression of vibrations is evidently more vital for the STM, long and narrow field-emission tips might not be satisfactory. Instead, we used solid metal rods of $1 \mathrm{~mm}$ diameter, and ground $90^{\circ}$ tips with a conventional grinding machine. This yielded overall tip radii of only some thousand angstroms to $1 \mu \mathrm{m}$, but with some rather sharp minitips. The extreme sensitivity of the tunnel current on gap width then selects the longest of the minitips for operation of the STM. The lateral resolution could be increased further by gently touching the surface with the tip and subsequently retracting it. This "mini-spot-welding" procedure created very fine tips, such that monoatomic steps could be resolved within $10 \AA$ laterally.

Scanning the tunnel tip at constant tunnel current implies $\varphi^{1 / 2} s=$ const. Thus, the $z$ displacement of the tunnel tip gives the surface topography only for constant work function $\varphi$, and therefore constant gap width $s$, as shown in Fig. 1 at $A$. On the other hand at $B$, the $z$ displacement is caused by a change of work function on a structureless part of the surface. However, true surface structures and work-function-mimicked structures can be separated by modulating the gap width $s$ while scanning, at a frequency higher than the cutoff frequency of the control unit. In a simple situation, as depicted in Fig. 1, the modulation signal gives the square root of the work function $\psi^{1 / 2}=\Delta\left(\ln J_{T}\right) / \Delta s$, directly, $A$ in Eq. (1) being nearly 1. For general surface topographies, and work-function profiles, the separation process becomes rather involved. Then, the modulation $\Delta s$ of the gap with $s$ is no longer equal to the length modulation $\Delta z$ of the piezodrive $P_{z}$. Essentially, $\Delta s=\Delta z \cos \phi$, where $\phi$ is the angle between the tunnel-surface element and the $z$ direction. In turn, the modulation signal is no longer constant at true surface structures even for constant work function $\varphi$. However, since $V_{p}$ and the modulation signal contain $\varphi$ and $s$ in a different way, their separation is, in principle, still possible even for involved structures and work-function profiles. In the following, we present topographic pictures of (110) surfaces of $\mathrm{CaIrSn}_{4}$ and $\mathrm{Au}$. Work-function profiles have not yet been studied in detail. They were used rather to get an overall picture of the surface condition.

CaIrSn $n_{4}$ - The flux-grown single crystals exhibited shiny, natural faces after solving the remaining flux in $\mathrm{HCl}$. Solvent etching probably stops at Ir layers, which appear to be rather 
inert. ${ }^{5}$ Therefore, they were good candidates for testing the operation of the STM at moderate vacuum $\left(\approx 10^{-6}\right.$ Torr $)$. Figure 2(a) shows a STM picture of a (110) surface obtained at room temperature without further surface treatment. We take the large flat parts (flat on an atomic scale) as an indication for a weak and homogeneous surface contamination. (No provision for simultaneous recording of work function and topography existed at the time of these experiments.) The pronounced structure on the left is the beginning of a growth spiral. Such spirals could be observed with both light and scanning electron microscopes. In the flat region, some monoatomic steps are clearly seen. Two scans with monoatomic, double-atomic, and triple-atomic steps are shown in Fig. 2(b). From all the steps observed, we obtained $6.7 \AA$ as the average spac ing of the $\operatorname{Ir}(110)$ planes. The piezodrives were calibrated by relacing the tip and sample with capacitor plates, giving a sensitivity of $2.0( \pm 0.2)$ $\AA / \mathrm{V}$ in each direction. This step height agrees well with the $6.87 \AA$ inferred from crystallographic data. ${ }^{6}$ Moreover, the form of the large steps is in qualitative agreement with that expected from simple calculations: a relatively sharp edge at the beginning of the step and considerable
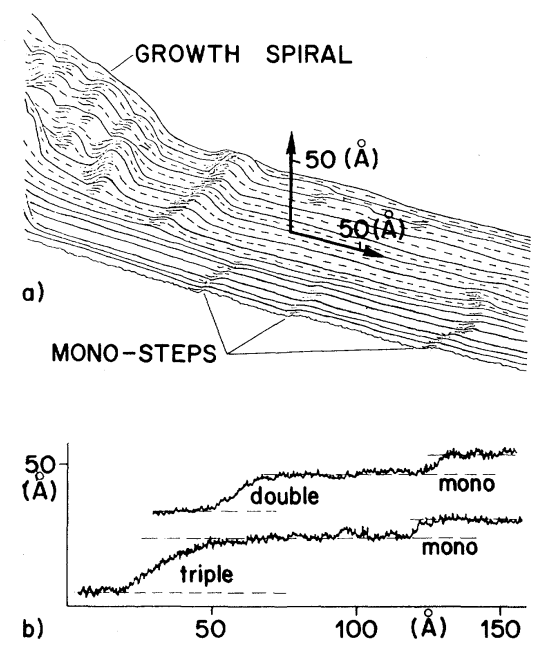

FIG. 2. Topography of a $\mathrm{CaIrSn}_{4}$ (110) surface. (a) Overall view of a flat part with single atomic steps (right) and start of a growth spiral (left). For better visualization of the topography of the surface, some additional lines have been interpolated (broken) between the smoothed scans. The bottom line is as measured. The distance between the scans is uncalibrated. (b) Two individual scans, exhibiting triple, double, and monoatomic steps. The broken lines indicate (110) faces, with the proper distance. smearing out at the end (as sketched in Fig. 1 at A).

$A u$.- The Au pictures were taken with a new, improved tunnel unit with considerably increased stability. The piezodrive material was calibrated in a conventional capacitance dilatometer within $2 \%$, giving an accuracy of the sensitivity of the whole piezodrive of about $5 \%$. The untreated (110) surface appeared structureless and mostly atomically flat. After Ar sputtering and subsequent annealing at $600^{\circ} \mathrm{C}$ in $(2$ to 7$) \times 10^{-10}$ Torr [a standard procedure for inducing reconstructions of $\mathrm{Au}$ (110) surfaces $^{7-9}$ ], the surface appeared gently corrugated in the [001] direction as shown in Fig. 3(a). The work function was practically constant. The modulated signal showed variations of the order of a percent which reflect the surface corrugation rather than a true variation of the work function, as explained above. Repetition of the cleaning procedure led to qualitatively the same result. The corrugation is not strictly periodic; it varies from 20 to $100 \AA$ in length and from some tenths to $2 \AA$ in height, but with only small local variation in

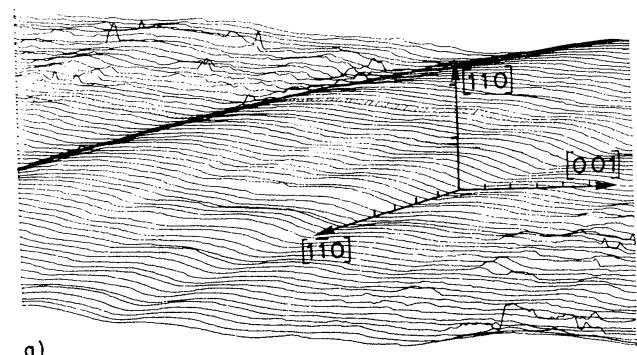

a)

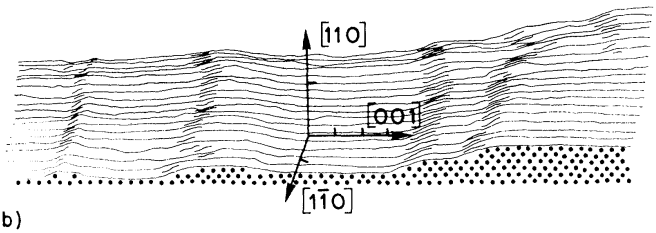

FIG. 3. Two examples of scanning tunneling micrographs of a $\mathrm{Au}$ (110) surface, taken at (a) room temperature, and (b) $300^{\circ} \mathrm{C}$ after annealing for $20 \mathrm{~h}$ at the same temperature (and essentially constant work function). The sensitivity is $10 \AA$ /div everywhere. Because of a small thermal drift, there is some uncertainty in the crystal directions in the surface. In (a), the surface is gently corrugated in the [001] direction, except for a step of four atomic layers ( $\cong 2$ atomic radii) along the [1]10] direction, as indicated by the discontinuity of the shaded ribbon. The steps in (b), which were always found along the [1 10$]$ direction, are visualized by the possible positions of the Au atoms (dots). 
periodicity and height. A small corrugation of about $100 \AA$ length in the [110] direction could be induced by rapidly cooling the sample to room temperature after annealing at $600{ }^{\circ} \mathrm{C}$. Atomic steps could not usually be observed, and the step of $6 \AA$ [ equal to four (110) spacings or two atomic radii] shown in Fig. $3(\mathrm{a})$ is an exception. How ever, double or monoatomic steps were easily found at $300{ }^{\circ} \mathrm{C}$ [ Fig. 3(b)]. An independent indication of an increasing step density with increasing temperature was recently obtained from an analysis of $\mathrm{He}$-diffraction line shapes for $\mathrm{Ni}(100) .^{10}$ Disorder along the [001] direction of $\mathrm{Au}(110)$ surfaces has been inferred from low-energy electron diffraction (LEED) experiments. ${ }^{8}$ The vary ing wavelength of the corrugation found in the present experiments induces such an anisotropic disorder. In view of the resolution demonstrated [see the steps in Fig. 3(b)], the corrugation is too smooth and flat to be explained in terms of some sequence of unrelaxed steps or a disordered $2 \times 1$ reconstruction of the missing-row type. ${ }^{8}$ It rather indicates a more continuous vertical displacement of the Au atoms. Surface buckling has been conjectured ${ }^{11}$ for the $\mathrm{Au}(100)$ surface as a consequence of a mismatch of a topmost hexagonal layer with the underlying fcc structure. Reconstructions of the (110) surface are subject to quite some controversy. In particular, spinpolarized LEED experiments seem to rule out any of the proposed models containing a mirror plane perpendicular to the [110] chains or twofold rotations. ${ }^{12}$ The distorted hexagonal topmost layer model ${ }^{13}$ is compatible with the symmetry requirements of the spin-polarized LEED results. Although the present experiment did not reveal the double periodicity in the [100] direction, some distorted hexagonal topmost-layer structure appears to be an attractive explanation for the long-wave buckling. Even more, nonobservation of the $2 \times 1$ structure in the present experiment could be considered as support of this model. However, it is not certain whether a $2 \times 1$ reconstruction was indeed present, although it had been previously observed in the same crystal by TEAMS experiments. ${ }^{7}$ Combined LEED and tunnel experiments are planned to clear this point. Finally, it is interesting to note that the step in Fig. 3(a) separates a smooth portion of the surface (on the right) from an atomically rough one.

In summary, we have shown that scanning tunneling microscopy yields a true three-dimensional topography of surfaces on an atomic scale, i.e., a resolution orders of magnitude better than scanning electron microscopy, with the possibility of extending it to work-function profiles (fourth dimension). The technique is nondestructive (energy of the tunnel "beam" $1 \mathrm{meV}$ up to 4 $\mathrm{eV}$ ), and uses fields down to three orders of magnitude less than field-ionization microscopy. The high current densities of $10^{3}$ to $10^{4} \mathrm{~A} / \mathrm{cm}^{2}$ appear to be no problem, and the technique has already been successfully extended to low-doped semiconductors. ${ }^{14}$ The significance of vacuum tunneling to surface studies and many other fields like space-resolved tunneling spectroscopy, microscopy of adsorbed molecules, and crystal growth, as well as for fundamental aspects of tunneling, especially in small geometries, is evident.

We thank R. Gambino and K. H. Rieder for providing the CaIrSn $\mathrm{Cn}_{4}$ and Aumples, respectively, H. R. Ott for calibrating the piezodrive material, B. Reihl and K. H. Rieder for discussions on surface aspects, and E. Courtens, K. A. Müller, and H. J. Scheel for their active interest in the STM.

\footnotetext{
${ }^{1}$ G. Binnig, H. Rohrer, Ch. Gerber, and E. Weibel, Physica (Utrecht) 107B + C, 1335 (1981), Proceedings of the Sixteenth International Conference on Low-Temperature Physics, Los Angeles, 19-25 August 1981.

${ }^{2}$ G. Binnig, H. Rohrer, Ch. Gerber, and E. Weibel, Appl. Phys. Lett. 40,178 (1981).

${ }^{3}$ R. D. Young, J. Ward, and F. Scire, Rev. Sci. Instrum. 43, 999 (1972).

${ }^{4}$ R. H. Fowler and L. Nordheim, Proc. Roy. Soc. London, Ser. A 119, 173 (1928); J. Frenkel, Phys. Rev. 36, 1604 (1930).

${ }^{5}$ R. Gambino, private communication.

${ }^{6}$ A. S. Cooper, Mater. Res. Bull. 15, 799 (1980).

${ }^{7}$ K. H. Rieder, T. Engel, and N. Garcia, in Proceedings of the Fourth International Conference on Solid Surfaces, and Third European Conference on Surface Science, Cannes 1980, Supplement to Revue Le Vide, Les Couche Minces, No. 201 (Societé Francaise du Vide, Paris, 1980), p. 861.

${ }^{8}$ D. Wolf, H. Jagodzinski, and M. Moritz, Surf. Sci. 77, 265, 283 (1978).

${ }^{9}$ J. R. Noonan and H. J. Davis, J. Vac. Sci. Technol. 16,587 (1979).

${ }^{10}$ K. H. Rieder and H. Wilsch, private communication.

${ }^{11}$ M. A. van Hove, R. J. Koestner, P. C. Stair, J. P. Bibérian, L. L. Kesmodel, I. Bartos, and G. A. Somorjai, Surf. Sci. 103, 181, 218 (1981).

${ }^{12}$ B. Reihl and B. I. Dunlap, Appl. Phys. Lett. 37, 941 (1980).

${ }^{13}$ E. Lang, K. Heinz, and K. Müller, Verh. Dtsch.
} 
Phys. Ges. 1, 278 (1978); E. Lang, private communication.

${ }^{14}$ G. Binnig and H. Rohrer, Europhys. Conf. Abstr. $\underline{6 A}$,
210 (1982), and Verh. Dtsch. Phys. Ges. $\underline{6}, 999$ (1982), and in Proceedings of the Sociéte Suisse de Physique Réunion de Printemps, 1982 (unpublished).

\title{
Physisorption Kinetics from Mean-Field Theory: Compensation Effect near Monolayer Coverage
}

\author{
E. Sommer (a) and H. J. Kreuzer \\ Theoretical Physics Institute, Department of Physics, University of Alberta, \\ Edmonton, Alberta T6G 2J1, Canada
}

(Received 25 February 1982)

\begin{abstract}
Based on a set of nonlinear rate equations with phonon-mediated transition rates calculated from mean field theory, the coverage-dependent isothermal desorption time for ${ }^{3} \mathrm{He}$ on graphite up to a coverage of 1.5 adlayers is determined. Prefactors in the Arrhenius parametrization drop by 2 orders of magnitude as the heat of adsorption decreases for increasing coverage, establishing a compensation effect in physisorption kinetics.
\end{abstract}

PACS numbers: $68.45 . \mathrm{Da}, 82.65 . \mathrm{My}$

A gas is said to physisorb onto the surface of a solid if the net interaction between a gas particle and the solid is accounted for by an effective surface potential $V_{s}(\overrightarrow{\mathrm{r}})$. which for an inert gas is well approximated by a sum $V_{s}(\overrightarrow{\mathbf{r}})=\sum_{i} V\left(\overrightarrow{\mathbf{r}}-\overrightarrow{\mathbf{r}}_{i}\right)$ where $V\left(\overrightarrow{\mathrm{r}}-\overrightarrow{\mathrm{r}}_{i}\right)$ is the two-body potential between a gas particle at $\vec{r}$ and a constituent particle of the solid at lattice site $\overrightarrow{\mathrm{r}}_{i}{ }^{1}$ Particles trapped into the bound states of $V_{s}(\vec{r})$ form the adsorbate. At very low (submonolayer) coverages $\theta$, we may neglect the interactions between the adparticles. However, as $\theta$ approaches unity and the average separation of gas particles in the adsorbate approaches that of a liquid, their mutual interaction potential plays a crucial role in ensuring saturation in a (mobile) fluid adsorbate or causing crystallization in the adsorbed film. We have recently developed a mean-field theory ${ }^{2,3}$ to describe an adsorbate of nonzero coverage in equilibrium with the gas phase. Such a theory is eminently suited to study the adsorption and desorption kinetics in such systems, a task that two-dimensional theories, ${ }^{4}$ in which the adsorbate is totally decoupled from the gas phase, have difficulty in tackling. For the study of adsorption kinetics it is important, for example, to know what changing environment additional particles arriving from the gas phase will experience as the coverage on the surface builds up. In a singleparticle picture this necessitates the construction of an effective coverage-dependent surface potential given by $V_{s}(\vec{r}, \theta)=V_{s}(\vec{r})+V_{m f}(\vec{r}, \theta)$, where $V_{s}(\overrightarrow{\mathrm{r}})$ is the interaction of a single gas particle with the solid, referred to from now on as the bare surface potential. $V_{m f}(\overrightarrow{\mathbf{r}}, \theta)$ is the potential arising from the mean field experienced by a gas particle in the presence of all other gas particles already in the surface region at a given coverage $\theta$. It can be calculated as a Slater average from the self-consistent solutions of the temperaturedependent Hartree-Fock equations

$$
\begin{aligned}
& {\left[-\left(\hbar^{2} / 2 m\right) d^{2} / d \overrightarrow{\mathbf{r}}^{2}+V_{s}(\overrightarrow{\mathbf{r}})-E_{\overrightarrow{\mathrm{j}}}\right] \psi_{\mathbf{i}}(r)+\sum_{\vec{j}} n_{j} \int d r^{\prime} V_{\text {eff }}\left(\left|\overrightarrow{\mathbf{r}}-\overrightarrow{\mathbf{r}}^{\prime}\right|\right) \psi_{\vec{j}^{\prime}} *\left(\overrightarrow{\mathbf{r}}^{\prime}\right)} \\
& \times\left[(2 s+1) \psi_{\vec{j}}\left(\overrightarrow{\mathbf{r}}^{\prime}\right) \psi_{\overrightarrow{\mathrm{i}}}(\overrightarrow{\mathbf{r}}) \mp \psi_{\vec{j}}(\overrightarrow{\mathbf{r}}) \psi_{\overrightarrow{\mathrm{i}}}\left(\overrightarrow{\mathbf{r}}^{\prime}\right)\right]=0,
\end{aligned}
$$

where $n_{\vec{j}}$ is the occupation function of the $\vec{j}$ th state; $s$ is the spin of the adsorbing gas particle, obeying Fermi-Dirac (minus sign) or Bose-Einstein (plus sign) statistics. $V_{\text {eff }}$ is the effective two-body interaction between gas particles whose short-range repulsion is suitably softened which, for gas particles obeying Fermi-Dirac statistics, can be done by employing Brueckner theory in the local density approximation. ${ }^{2,3}$ For mobile adsorbates the bare surface potential can be approximated by a function of the distance $z$ from the surface only, i.e., $V_{s}(\overrightarrow{\mathbf{r}}) \equiv V_{s}(z)$, so that an Ansatz $\psi \overrightarrow{\mathbf{i}}(\overrightarrow{\mathbf{r}})=\varphi \overrightarrow{\mathrm{i}}(z) \exp [i \overrightarrow{\mathrm{q}} \cdot \vec{\rho}]$ is justified $[\overrightarrow{\mathrm{r}}$ $=(\vec{p}, z) ; \vec{q}$ is the particle momentum in the sur face plane] reducing (1) cum grano salis to a one-dimensional theory for the wave functions $\varphi_{\overrightarrow{\mathrm{i}}}(z)$ and the energies $\epsilon_{i}=E_{\overrightarrow{\mathrm{i}}}-\hbar^{2} q^{2} / 2 m$ and re- 